\title{
Bio-complexes as supermolecules: towards design of idealized peptide-based ligands
}

\author{
Joanna Bojarska \\ Department of Chemistry, Technical University of Lodz, Poland \\ joanna.bojarska@p.lodz.pl
}

Modified amino acids and short peptides, as biologically active molecules and constituents of proteins, can be a golden remedy for diverse diseases, including viral infections, cancers or neurodegenerative disorders, due to their unique features, such as selectivity and specificity toward specific targets, that have been grinded, like a diamond, by evolution over a milion years. Peptides act as signalling entities via all domains of life and interfere with protein-protein interactions, which are indispensable in bio-processes. Advances in bio-nano-technological sciences and bio-informatics lead to overcome their poor ADMET profile. As consequence, these the simplests biomolecules are glimmer of hope for next generation, effective and safe theranostics [1]. From the point of view of the drug discovery, supramolecular hydrogen-bonding synthon concept [2] is promising tool in future design of idealized ligands, with effective binding, inside the protein pockets through matching synthonic functionalities (from corresponding libraries) to the model ligands [3]. Notably, the same synthons, structural units formed by synthetic operations requiring non-covalent interactions, are observed both in supramolecular systems of ligands and bio-complexes. The latter can be considered as supermolecule, which has been underestimated so far. The supramolecular studies of biomolecules, which cannot be mimicked by no other chemical compounds, are of prime importance. The idea of design and development of innovative and smart therapeutic peptide-based agents by utilizing of supramolecular synthon approach in ligand-protein complexes will be discussed in detail.

[1] Apostolopoulos, V., Bojarska, J., Chai, T.-T., Elnagdy, S., Kaczmarek, K., Matsoukas, J., et al. (2021). A Global Review on Short Peptides: Frontiers and Perspectives. Molecules 26, 430-475. doi:10.3390/molecules26020430

[2] Bojarska, J., Kaczmarek, K., Zabrocki, J., and Wolf, W.M. (2018a). Supramolecular Chemistry of Modified Amino Acids and Short Peptides. In Advances in Organic Synthesis; A. Rahman, Ed.; Bentham Science Publishers Ltd.: Sharjah, UAE, Volume 11, pp. 43-107. doi: $10.2174 / 9781681087474118110004$

[3] Spackman, P. R., Yu, L. J., Morton, C. J., Parker, M. W., Bond, C. S., Spackman, M. A., et al. (2019). Bridging Crystal Engineering and Drug Discovery by Utilizing Intermolecular Interactions and Molecular Shapes in Crystals. Angew. Chem. 131, 16936-16940. doi:10.1002/ange.201906602

Keywords: short peptides; supermolecules; supramolecular synthons 